\title{
COMPARAÇÃO DE MODELOS MATEMÁTICOS PARA DESCRIÇÃO DA CINÉTICA DE SECAGEM EM CAMADA FINA DE SEMENTES DE FEIJÃO
}

\author{
Paulo Cesar Afonso Júnior ${ }^{1}$ \& Paulo Cesar Corrêa ${ }^{2}$
}

\begin{abstract}
RESUMO
Para fornecer informações sobre o processo de secagem de sementes de feijão, este trabalho foi desenvolvido objetivando-se determinar as curvas de secagem em camada fina, para quatro níveis de temperatura do ar de secagem $\left(35,40,45\right.$ e $\left.50^{\circ} \mathrm{C}\right)$ e três níveis de teor de umidade inicial $(0,206 ; 0,373 \mathrm{e}$ 0,596 b.s.). Os testes de secagem foram realizados com três repetições, utilizando-se um secador experimental com fluxo de ar aproximadamente constante, de $10 \mathrm{~m}^{3} \mathrm{~min}^{-1} \mathrm{~m}^{-2} \mathrm{e}$ os resultados da secagem foram avaliados ajustando-se os seguintes modelos matemáticos: Thompson, Exponencial, Page e Difusão. Os resultados obtidos, neste trabalho, permitiram concluir que a equação proposta por Page é a que melhor representa os dados experimentais.
\end{abstract}

Palavras-chave: secagem, camada fina, Phaseolus vulgaris

\section{COMPARISON OF MATHEMATICAL MODELS FOR DESCRIPTION OF THE KINETICS OF THIN-LAYER DRYING OF BEAN SEEDS}

\begin{abstract}
To provide information about the drying process of bean seeds, this study was conducted with the objective of determining the thin-layer drying curves, for four levels of temperature of drying air $\left(35,40,45\right.$ and $\left.50^{\circ} \mathrm{C}\right)$ and three levels of initial moisture content $(0.206,0.373$ and 0.596 d.b.). The drying tests were accomplished with three replications, using an experimental dryer with a constant air flow of approximately $10 \mathrm{~m}^{3} \mathrm{~min}^{-1} \mathrm{~m}^{-2}$. The results of the drying were adjusted to the following mathematical models: Thompson, Exponential, Page and Diffusion. The results obtained in this study lead to the conclusion that the equation proposed by Page best represented the experimental data.
\end{abstract}

Key words: drying, thin-layer, Phaseolus vulgaris

\section{INTRODUÇÃO}

Com a expansão da cultura do feijão nas principais regiões produtoras do país, técnicas modernas de secagem vêm sendo desenvolvidas para atender às crescentes necessidades dos produtores, com o objetivo de simplificar e reduzir os custos associados ao processo produtivo. No Brasil, a produção de feijão cresceu de 2.006.005 t em 1987, com uma área colhida de
5.221.794 ha, para 2.822.340 t em 1996, com uma área colhida de 4.944.699 ha devido, principalmente, à adoção de um conjunto de tecnologias e insumos mais modernos.

A produção de grãos ou sementes com elevada qualidade requer, entre outras recomendações, que o produto seja colhido sadio e antecipadamente, visando minimizar as perdas ocasionadas no campo pelo ataque de insetos, doenças e microrganismos; desta maneira e devido ao teor de umidade

\footnotetext{
${ }^{1}$ Eng. Agrícola, M.Sc.., Doutorando em Eng. Agrícola, DEA-UFV, CEP 36571 - 000, Viçosa, MG, E-mail: pjunior@alunos.ufv.br

${ }^{2}$ Eng. Agrônomo, D.S., Prof. Adjunto, DEA-UFV, CEP 36571 - 000, Viçosa, MG, Fone: (031) 891 2270, Fax: (031) 891 1943, E-mail: copace@ mail.ufv.br
} 
elevado por ocasião da colheita, a secagem constitui uma das operações de primordial importância entre as técnicas envolvidas na conservação das qualidades desejáveis de produtos de origem vegetal.

A secagem de produtos agrícolas pode ser definida como um processo simultâneo de transferência de calor e massa entre o produto e o ar de secagem, que consiste na remoção da umidade excessiva contida no interior do grão por meio de evaporação, geralmente causada por convecção forçada de ar aquecido, de modo a permitir a manutenção de sua qualidade durante o armazenamento, por longos períodos de tempo.

O estudo de sistemas de secagem, seu dimensionamento, otimização e a determinação da viabilidade de sua aplicação comercial, podem ser feitos por simulação matemática. Para a simulação, cujo princípio se fundamenta na secagem de sucessivas camadas delgadas do produto, utiliza-se um modelo matemático que representa satisfatoriamente a perda de umidade do produto durante o período de secagem.

$\mathrm{Na}$ literatura, encontram-se vários métodos propostos para se analisar a secagem em camada fina de produtos higroscópicos: os teóricos, os semiteóricos e os empíricos.

Os modelos de secagem baseados na teoria de difusão de líquido têm sido preferidos por muitos pesquisadores na área de secagem de alimentos e grãos (Igbeka, 1982; Mulet et al., 1989; Sereno \& Medeiros, 1990; Queiroz \& Nebra, 1997) embora existam algumas suposições a serem consideradas para aplicação desses modelos, como: a redução de volume é desprezível; não há efeito de capilaridade; os corpos entram em equilíbrio térmico com ar instantaneamente e os efeitos da transferência de energia e massa de um corpo para outro são desprezíveis; entretanto, devido às limitações de ordem prática, quando utilizadas para produtos biológicos estas suposições são normalmente consideradas satisfeitas.

De acordo com Brooker et al. (1992) a variação de umidade com o tempo de secagem para materiais homogêneos com coeficiente de difusão constante é representada pela seguinte equação:

$$
\frac{\partial \mathrm{U}}{\partial \mathrm{t}}=\mathrm{D}\left[\frac{\partial^{2} \mathrm{U}}{\partial \mathrm{r}^{2}}+\frac{\mathrm{c}}{\mathrm{r}} \cdot \frac{\partial \mathrm{U}}{\partial \mathrm{r}}\right]
$$

em que:

U - teor de umidade, decimal base seca

D - coeficiente de difusão de líquido, $\mathrm{m}^{2} \mathrm{~s}^{-1}$

$\mathrm{t}$ - tempo, s

c $\quad$ - 0 para corpos planos, 1 para corpos cilíndricos e 2 para corpos esféricos

r - coordenada espacial, dependendo da forma geométrica, $\mathrm{m}$

Crank, apud Brooker et al. (1992) mostra a solução analítica para produtos com forma geométrica esférica:

$$
\mathrm{RU}=\frac{\mathrm{U}-\mathrm{Ue}}{\mathrm{Uo}-\mathrm{Ue}}=\frac{6}{\pi^{2}} \sum_{\mathrm{n}=1}^{\infty} \frac{1}{\mathrm{n}^{2}} \exp \left[-\frac{\mathrm{n}^{2} \pi^{2} \cdot \mathrm{D} \cdot \mathrm{t}}{9}\left(\frac{3}{\mathrm{R}}\right)^{2}\right]=\frac{6}{\pi^{2}} \sum_{\mathrm{n}=1}^{\infty} \frac{1}{\mathrm{n}^{2}} \exp \left[-\mathrm{n}^{2} \cdot \alpha \cdot \mathrm{t}\right](2)
$$

em que:

RU - razão de umidade do produto, adimensional

Uo - teor de umidade inicial, decimal base seca

Ue - teor de umidade de equilíbrio, decimal base seca
$\mathrm{R}$ - distância radial até a superfície, $\mathrm{m}$

a $\quad-\left(p^{2} \cdot D / 9\right) \cdot(3 / R)^{2}$

Observa-se que a solução analítica dessa equação apresenta-se na forma de uma série infinita e, portanto, o número finito de termos (n) no truncamento poderá determinar a precisão dos resultados.

Os métodos semi-empíricos oferecem um compromisso entre a teoria e a aplicação sem, entretanto, refletirem toda a complexidade do fenômeno. Baseiam-se, geralmente, na lei de resfriamento de Newton aplicada à transferência de massa, presumindo-se que, durante a secagem, as condições sejam isotérmicas e que a transferência de umidade se restrinja à superfície do produto (Syarief et al., 1984).

Lewis, apud Brooker et al. (1992) sugeriu que, durante o processo de secagem de materiais higroscópicos porosos no período de taxa de secagem decrescente, a mudança da umidade fosse proporcional à diferença instantânea entre o teor de umidade do material e o teor de umidade do material, quando em condições de equilíbrio com o ar de secagem, ou seja:

$$
\frac{\partial \mathrm{U}}{\partial \mathrm{t}}=-\mathrm{k}(\mathrm{U}-\mathrm{Ue})
$$

em que:

$\mathrm{k}$ - constante de secagem, $\mathrm{s}^{-1}$, enquanto outros símbolos foram definidos anteriormente

Assumindo k como uma constante e integrando a Eq. 3 para incrementos de tempo de 0 at, e para teores de umidade decrescentes (U) a partir do teor de umidade inicial (Uo), tem-se que:

$$
\frac{\mathrm{U}-\mathrm{Ue}}{\mathrm{Uo}-\mathrm{Ue}}=\mathrm{RU}=\exp (-\mathrm{k} \cdot \mathrm{t})
$$

Page, citado por Sharma et al. (1982) com base na Eq. 4, conhecida como modelo exponencial, propôs um modelo empírico para descrever a secagem de grãos em camada fina, da seguinte forma:

$$
\frac{\mathrm{U}-\mathrm{Ue}}{\mathrm{Uo}-\mathrm{Ue}}=\mathrm{RU}=\exp \left(-\mathrm{k} \cdot \mathrm{t}^{\mathrm{n}}\right)
$$

em que n representa um parâmetro adimensional de secagem.

Bruce (1985) afirma que o modelo proposto por Page descreve melhor o processo de secagem de produtos agrícolas que o modelo exponencial.

Os parâmetros dos modelos de secagem em camada fina são calculados em função das variáveis que influenciam a taxa de secagem. Em geral, a temperatura é uma variável sempre presente nos modelos que descrevem o processo de secagem (Dung et al., 1980; Bala \& Woods, 1992). Outra variável normalmente associada aos parâmetros de secagem é o teor de umidade inicial do produto (White et al., 1981; Soares, 1986).

Thompson et al. (1968) desenvolvendo um modelo de simulação para secagem de grãos em camada estacionária, propuseram uma equação de secagem em camada fina totalmente empírica, para descrever o processo:

$$
\mathrm{t}=\mathrm{A} \cdot \ln (\mathrm{RU})+\mathrm{B} \cdot[\ln (\mathrm{RU})]^{2}
$$

em que os parâmetros A e B são funções da temperatura do ar 
de secagem. Este modelo tem sido muito utilizado para auxiliar nas simulações de secagem de milho.

Ante o exposto, o presente trabalho teve como objetivo determinar, experimentalmente, as curvas de secagem para sementes de feijão e ajustar diferentes modelos matemáticos aos dados experimentais, em função da temperatura do ar de secagem e do teor de umidade inicial do produto.

\section{MATERIAL E MÉTODOS}

O presente trabalho foi realizado no Laboratório de Pré-Processamento e Armazenagem de Produtos Agrícolas do Departamento de Engenharia Agrícola, pertencente ao Centro de Ciências Agrárias da Universidade Federal de Viçosa.

Foram utilizadas sementes de feijão (Phaseolus vulgaris L.) cultivar "Ouro Negro 1992", colhidas e debulhadas manualmente, com teores iniciais de umidade de 0,206; 0,373 e 0,596 b.s., formando três lotes distintos de sementes.

$\mathrm{O}$ teor de umidade inicial das sementes foi determinado pelo método padrão da estufa, $105 \pm 3{ }^{\circ} \mathrm{C}$, durante $24 \mathrm{~h}$, com três repetições, de acordo com as regras para análise de sementes (Brasil, 1992).

Os tratamentos de secagem constituíram-se de um esquema fatorial $4 \times 3$, com quatro níveis de temperatura $\left(35,40,45\right.$ e $\left.50^{\circ} \mathrm{C}\right)$ e três níveis de teores de umidade inicial $(0,206 ; 0,373$ e 0,596 b.s.) num delineamento inteiramente casualizado, com três repetições. Para cada tratamento de secagem foram utilizados em torno de $500 \mathrm{~g}$ de sementes por repetição.

Os testes de secagem foram realizados utilizando-se um secador experimental com ar de secagem fornecido por um ventilador axial. $\mathrm{O}$ ar com velocidade constante era conduzido até o plênum, onde era distribuído em três bandejas removíveis contendo as amostras de sementes.

$\mathrm{O}$ ar foi aquecido por meio de resistências elétricas, enquanto a temperatura foi controlada com o auxílio de um transformador variador de tensão de 0 a $140 \mathrm{~V}$, conectado, em série, às resistências elétricas. Os valores de temperatura foram obtidos por meio de um termômetro de mercúrio, cujo bulbo foi instalado imediatamente abaixo da camada de sementes.

O fluxo de ar foi determinado a partir da velocidade do ar de secagem utilizando-se um anemômetro de lâminas rotativas e controlado por meio de um diafragma, adaptado à entrada de ar do ventilador e mantido constante para todos os tratamentos de secagem, em aproximadamente $10 \mathrm{~m}^{3} \mathrm{~min}^{-1} \mathrm{~m}^{-2}$.

Para o cálculo da razão de umidade (RU) calculou-se a umidade de equilíbrio higroscópico pela equação modificada por Henderson, obtida por Bach (1979) para feijão preto:

$$
\mathrm{Ue}=\left[\frac{\ln (1-\mathrm{UR})}{\left(-8,0707 \times 10^{-16}\right) \cdot(\mathrm{T}+273,15)^{5,2304}}\right]^{1 / 1,7692}
$$

em que:

Ue - teor de umidade de equilíbrio, \% base seca

UR - umidade relativa do ar de secagem, decimal

$\mathrm{T}$ - temperatura do ar de secagem, ${ }^{\circ} \mathrm{C}$.

Para cada tratamento de secagem, a umidade relativa do ar secante era calculada por meio de um programa computacional desenvolvido a partir de equações psicrométricas (GRAPSI) e um psicrômetro, instalado na entrada de ar do ventilador.
Durante a operação de secagem foram realizadas pesagens periódicas das amostras, até atingir-se o teor de umidade final preestabelecido de 0,124 b.s.

Para o ajuste dos modelos matemáticos aos dados experimentais de secagem, realizou-se análise de regressão não linear, pelo método Quasi-Newton, utilizando-se o programa computacional STATISTICA 5.0. Estimaram-se os valores dos parâmetros dos modelos, em função das variáveis independentes temperatura do ar de secagem e umidade inicial do produto.

Para relacionar os parâmetros dos modelos estudados com as variáveis temperatura e umidade inicial, optou-se pelo modelo estatístico apresentado a seguir:

$$
Y i j=b_{0}+b_{1} T i+b_{2} T^{2}+b_{3} U j+b_{4} U j^{2}+b_{5}(T . U) i j+E i j
$$

em que:

Yij - valores observados dos parâmetros, para o modelo em estudo, na temperatura i e teor de umidade inicial $\mathrm{j}$

bo - constante da regressão

be - coeficiente da regressão, $\mathrm{e}=1, \ldots, 5$

$\mathrm{Ti}$ - temperatura do ar de secagem, ${ }^{\circ} \mathrm{C}$

$\mathrm{Uj} \quad$ - teor de umidade inicial, decimal b.s.

Eij - erro aleatório.

A partir desse modelo foram testados outros modelos, eliminando-se o efeito quadrático $\left(\mathrm{T}^{2}\right.$ ou $\left.\mathrm{U}^{2}\right)$, a interação (T.U) e um dos fatores ( $\mathrm{T}$ ou $\mathrm{U}$ ).

O erro médio relativo (P) e o erro médio da estimativa (SE) para cada modelo, foram calculados conforme descrito a seguir:

$$
\begin{aligned}
& \mathrm{P}=\frac{100}{\mathrm{n}} \cdot \sum \frac{|\mathrm{Y}-\hat{\mathrm{Y}}|}{\mathrm{Y}} \\
& \mathrm{SE}=\sqrt{\frac{\sum(\mathrm{Y}-\hat{\mathrm{Y}})^{2}}{\mathrm{GLR}}}
\end{aligned}
$$

em que:

$$
\begin{array}{ll}
\mathrm{n} & \text { - número de observações } \\
\mathrm{Y} & \text { - valor observado experimentalmente } \\
\hat{\mathrm{Y}} & \text { - valor calculado pelo modelo } \\
\mathrm{GLR} & \text { - graus de liberdade do modelo. }
\end{array}
$$

$\mathrm{O}$ grau de ajuste do modelo aos dados experimentais baseou-se na magnitude do coeficiente de determinação ajustado, na significância dos coeficientes de regressão, na magnitude do erro médio relativo e do erro médio da estimativa e na verificação de falta de ajuste do modelo estatístico.

\section{RESULTADOS E DISCUSSÃO}

Na Tabela 1 está apresentado o resumo dos modelos ajustados por meio de regressão não linear aos dados experimentais de secagem de sementes de feijão em camada fina, considerando-se a temperatura do ar de secagem e o teor de umidade inicial do produto como variáveis independentes.

Observa-se que a equação com base no modelo de Page foi a que melhor se ajustou aos dados observados de razão de umidade do feijão, com coeficiente de determinação para os parâmetros k e n de 0,9912 e 0,9558 , respectivamente, e porcentagem média de desvio de 2,6361, apresentando melhor 
Tabela 1. Parâmetros das equações ajustadas para se calcular a razão de umidade (RU) de sementes de feijão em camada fina, em função da temperatura do ar de secagem $(\mathrm{T})$ e do teor de umidade inicial (U) com os respectivos coeficientes de determinação ( $\left.\mathrm{R}^{2}\right)$, porcentagem média de desvio $(\mathrm{P})$ e erro médio da estimativa (SE)

\begin{tabular}{|c|c|c|c|}
\hline Modelo e Parâmetros & $\mathrm{R}^{2}$ & $\mathrm{P}$ & SE \\
\hline $\begin{array}{l}\text { Page } \\
k=0,4460-0,0236^{*} \cdot T+0,0003^{*} \cdot T^{2}+1,0833^{*} \cdot U-0,6902^{*} \cdot U^{2} \\
n=1,0591-0,0056^{*} \cdot T-1,6569^{*} \cdot U+1,4230^{*} \cdot U^{2}+0,0121^{*} \cdot T \cdot U\end{array}$ & $\begin{array}{l}0,9912 \\
0,9558\end{array}$ & 2,6361 & 0,3153 \\
\hline $\begin{array}{l}\text { Difusão (8 Termos) } \\
\alpha=0,2665-0,0128^{*} \cdot T+0,0002^{*} \cdot T^{2}+0,0627^{*} \cdot U+0,0043^{*} . \mathrm{T} . U\end{array}$ & 0,9875 & 4,0417 & 0,5579 \\
\hline $\begin{array}{l}\text { Thompson } \\
\begin{array}{l}\mathrm{A}=-11,4460+0,1762^{*} . \mathrm{T}+18,4420^{*} \cdot \mathrm{U}-6,9674^{*} \cdot \mathrm{U}^{2}-0,2412^{*} . \mathrm{T} . \mathrm{U} \\
\mathrm{B}=-16,4561+1,3717^{*} . \mathrm{T}-0,0161^{*} \cdot \mathrm{T}^{2}-31,4268^{*} \cdot \mathrm{U}+23,5601^{*} \cdot \mathrm{U}^{2}\end{array}\end{array}$ & $\begin{array}{l}0,9672 \\
0,9649\end{array}$ & 12,2191 & 16,7623 \\
\hline $\begin{array}{l}\text { Exponencial } \\
\mathrm{k}=0,5059-0,0239 * \cdot \mathrm{T}+0,0003^{*} \cdot \mathrm{T}^{2}+0,0798^{\text {n.s. }} \cdot \mathrm{U}+0,4322 * \cdot \mathrm{U}^{2}\end{array}$ & 0,9884 & 17,4339 & 1,7605 \\
\hline
\end{tabular}

* - Significativo nível de $1 \%$ de probabilidade pelo teste "t"

n.s. - Não significativo

A. Page

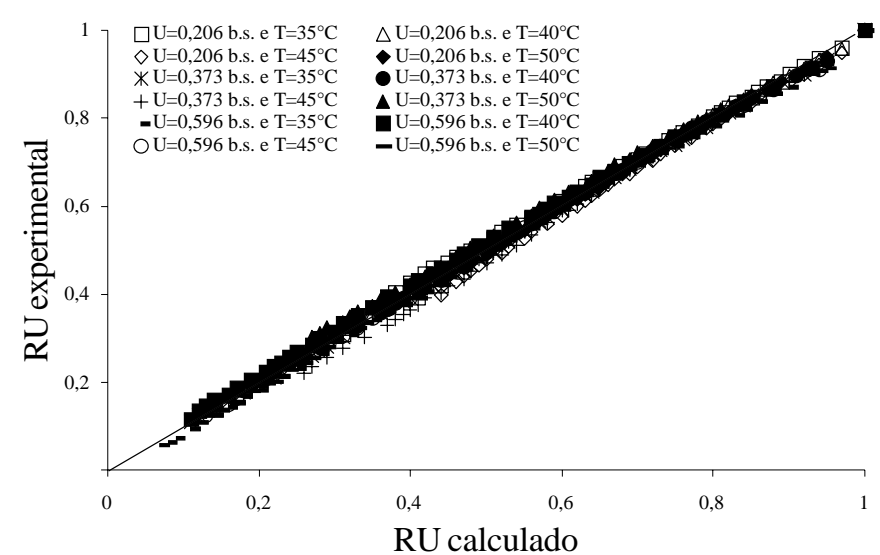

C. Thompson

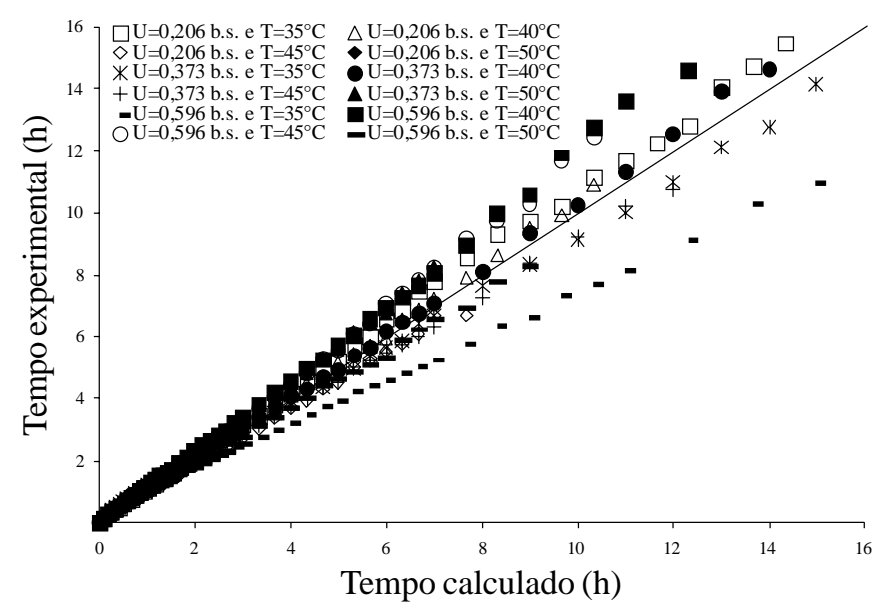

B. Difusão

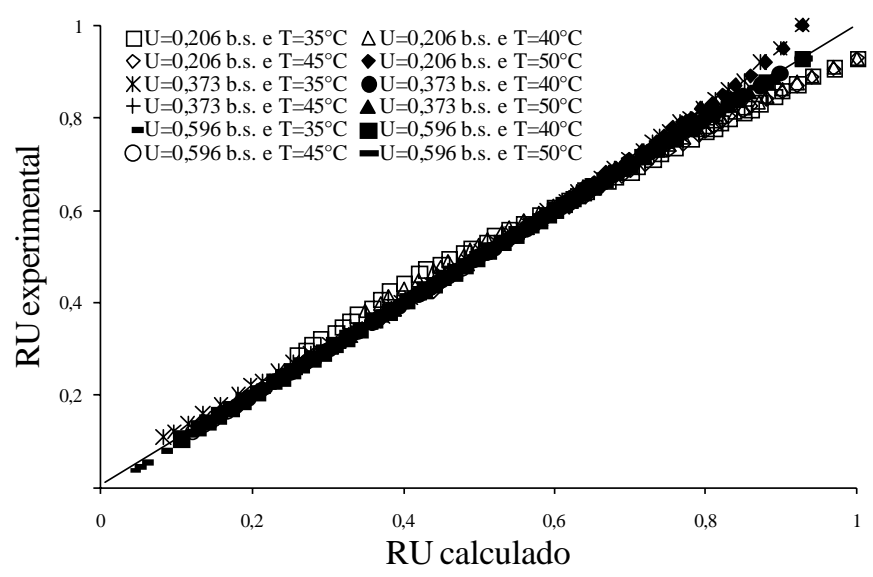

D. Exponencial

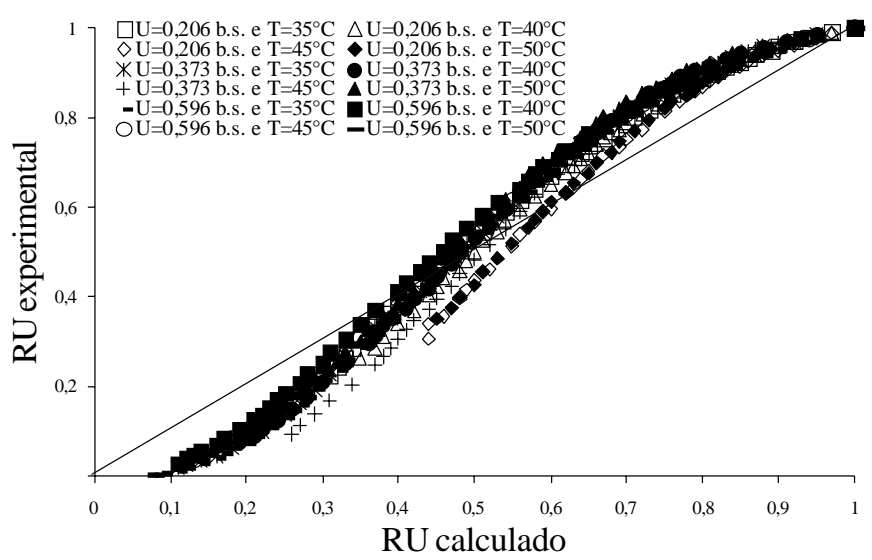

Figura 1. Relação entre valores experimentais e calculados para secagem de sementes de feijão, com o emprego do modelo de (A) Page, (B) Difusão, (C) Thompson e (D) Exponencial

comportamento para representar a secagem de sementes de feijão em camada delgada; entretanto, a análise dos dados da Tabela 1 indica que o modelo de secagem baseado na teoria de difusão também se mostrou adequado para fornecer estimativas satisfatórias da taxa de secagem de sementes de feijão.
Na Figura 1 estão apresentados os gráficos de correspondência para os quatro modelos matemáticos de secagem das sementes de feijão. Esses gráficos indicam o ajustamento das equações de secagem, ou seja, a dispersão dos dados experimentais em relação aos dados estimados, considerando-se todos os dados obtidos durante o experimento. 
Verifica-se que, para o modelo de Thompson, houve tendência mais acentuada de dispersão dos valores calculados no período final do processo de secagem, principalmente para as sementes com teor de umidade mais elevado (0,596 b.s.). Observa-se, ainda, que o modelo Exponencial foi o que apresentou os maiores desvios entre os valores experimentais e calculados para representação do processo de secagem de sementes de feijão em camada fina.

Os resultados encontrados neste trabalho, mostrando que a equação de Page foi a que apresentou melhor ajuste aos dados experimentais de secagem, estão de acordo com os resultados encontrados por diversos autores para diferentes produtos agrícolas (White et al., 1981; Soares, 1986; Ramos et al., 1993/ 1994; Christ, 1996).

Embora várias teorias tenham sido propostas para predizer o comportamento da secagem de grãos e sementes, na maioria das vezes as relações semi-empíricas e empíricas têm-se mostrado como melhores opções para predizer o processo de secagem, apesar de sua validade estar restrita às condições sob as quais os dados experimentais foram obtidos.

\section{CONCLUSÕES}

Os resultados obtidos neste trabalho permitiram concluir que:

1. A equação proposta por Page é a que melhor representa os dados experimentais, quando comparada com as equações de Thompson, Exponencial e de Difusão, utilizando-se oito termos da série.

2. Os parâmetros dos modelos estudados foram significativamente afetados pela temperatura do ar de secagem e teores de umidade inicial das sementes.

\section{AGRADECIMENTOS}

Os autores agradecem à FINEP/RECOPE, pelo auxílio financeiro.

\section{REFERÊNCIAS BIBLIOGRÁFICAS}

BACH, D.B. Curvas de equilíbrio higroscópico de feijão-preto (Phaseolus vulgaris L.). Viçosa: Universidade Federal de Viçosa, 1979. 39p. Dissertação Mestrado

BALA, B.K.; WOODS, J.L. Thin layer drying models for malt. Journal of Food Engineering, Barking, v.16, n.1, p.239-249, 1992.

BRASIL, Ministério da Agricultura e Reforma Agrária. Regras para análise de sementes. Brasília: DNDV/CLAV, 1992.365p.
BROOKER, D.B.; BAKKER-ARKENA, F.W.; HALL, C.W. Drying and storage of grains and oilseeds. New York: AVI Publishing Company, 1992. 450p.

BRUCE, D.M. Exposed-layer barley drying: Three models fitted to new data up to $150{ }^{\circ} \mathrm{C}$. Journal of Agricultural Engineering Research, London, v.32, n.4, p.337-347, 1985.

CHRIST, D. Curvas de equilíbrio higroscópico e de secagem da canola (Brassica napus L. var. oleifera) e efeito da temperatura e da umidade relativa do ar de secagem sobre a qualidade das sementes. Viçosa: Universidade Federal de Viçosa, 1996. 50p. Dissertação Mestrado

DUNG, N.V.; FOWLER, R.T.; BOWREY, R.G. Variables affecting the drying rate of paddy rice. Food Technology in Australia, Sidney, v.32, n.12, p.604-606, 1980.

IGBEKA, J.C. Simulation of moisture movement during drying a starchy food product-cassava. Journal of Food Technology, London, v.17, n.1, p.27-36, 1982.

MULET, A.; BERNA, A.; ROSSELO, C. Drying of carrots: Drying models. Drying Technology, New York, v.7, n.3, p.537-557, 1989.

QUEIROZ, M.R.; NEBRA, S.A. Soluções analíticas e numéricas do modelo de difusão à secagem de bananas. Engenharia Agrícola, Jaboticabal, v.17, n.1, p.74-83, 1997.

RAMOS, A.M.; PEREIRA, J.A.M.; QUEIROZ, D.M. Equações de secagem de trigo em camada fina e determinação dos coeficientes de difusão de líquido. Revista Brasileira de Armazenamento, Viçosa, v.18-19, n.1-2, p.43-47, 1993/1994.

SERENO, A.M.; MEDEIROS, G.L. Simplified model for the prediction of drying rates for foods. Journal of Food Engineering, Barking, v.12, n.1, p.1-11, 1990.

SHARMA, A.D.; KUNZER, O.R.; TOLLEY, H.D. Rough rice drying as a two-compartment model. Transaction of the ASAE, St. Joseph, v.25, n.1, p.221-224, 1982.

SOARES, J.B. Curvas de secagem em camada fina e propriedades físicas de soja (Glicine max L.). Viçosa: Universidade Federal de Viçosa, 1986. 91p. Dissertação Mestrado

SYARIEF, A.M.; MOREY, R.V.; GUSTAFSON, R.J. Thin-layer drying rate of sunflower seed. Transaction of the ASAE, St. Joseph, v.27, n.1, p.195-200, 1984.

THOMPSON, T.L; PEART, R.M.; FOSTER, G.H. Mathematical simulation of corn drying - A new model. Transaction of the ASAE, St. Joseph, v.11, n.4, p.582-586, 1968.

WHITE, G.M.; BRIDGES, T.C.; LOEWER, O.J.; ROSS, I.J. Thin-layer drying model for soybeans. Transaction of the ASAE, St. Joseph, v.24, n.6, p.1643-1646, 1981. 